\title{
UTILIZAÇÃO DE TEORIAS DE ENFERMAGEM NA SISTEMATIZAÇÃO DA PRÁTICA CLÍNICA DO ENFERMEIRO: REVISÃO INTEGRATIVA
}

\section{USE OF NURSING THEORIES TO THE SYSTEMATIZATION OF THE PRACTICE OF THE NURSE: INTEGRATIVE LITERATURE REVIEW}

\section{USO DE TEORÍAS DE ENFERMERÍA PARA LA SISTEMATIZACIÓN DE LA PRÁCTICA DEL ENFERMERO: REVISIÓN INTEGRADORA}

\author{
Vívian Mayara da Silva Barbosa ${ }^{1}$, John Victor dos Santos Silva ${ }^{2}$
}

\begin{abstract}
RESUMO
Objetivo: analisar como os enfermeiros utilizam teorias de enfermagem na sistematização de sua prática clínica. Metodologia: revisão integrativa da literatura, realizada nas bases de dados LILACS, BDENF e SciELO, de onde foram selecionados 19 artigos que compuseram a amostra. Resultados: em $79 \%$ dos artigos analisados foi enfatizada a necessidade de utilizar um modelo teórico para implementar o Processo de Enfermagem, sendo as mais utilizadas a Teoria da Adaptação de Callista Roy e a de Jean Watson, ambas utilizadas para a implementação da Sistematização da Assistência de Enfermagem em 15,7\% da amostra. Dentre os ambientes foco de sua utilização, destaca-se o hospitalar com 52\%, a atenção primária com $21 \%$ e o domiciliar com $15 \%$. Conclusão: na enfermagem brasileira, que apesar de ser considerada uma das maiores produtoras de publicações científicas na atualidade, contempla-se ainda uma baixa utilização de teorias de enfermagem aliadas à sistematização do seu fazer específico.
\end{abstract}

Descritores: Enfermagem; Teoria de Enfermagem; Processo de Enfermagem.

\begin{abstract}
Objective: to analyze how nurses use the nursing theories to the systematization of their clinical practice. Methodology: integrative review of literature. Searches were held in the databases BDENF, LILACS and SciELO. Results: $79 \%$ of the articles analyzed was emphasized the need to use a theoretical model for implementing the nursing process, being the most used the theory of adaptation of Callista Roy and Jean Watson, both used for the Nursing Process Performance implementation in $15.7 \%$ of the sample. One of the focus of its use, with $52 \%$, the hospital primary care with $21 \%$ and $15 \%$ with home. Conclusion: although the brazilian nursing be considered one of the largest producers of scientific publications at present, includes a low utilization of nursing theories combined with the systematization of your do.
\end{abstract}

Keywords: Nursing; Nursing Theory; Nursing Process.

\footnotetext{
${ }^{1}$ Enfermeira. Mestranda em Enfermagem pela Universidade Federal de Alagoas.

${ }^{2}$ Acadêmico de Enfermagem pela Universidade Estadual de Ciências da Saúde de Alagoas. Presidente da Liga Acadêmica Interdisciplinar de Saúde Mental (LAISME-UNCISAL).
} 


\section{RESUMEN}

Objetivo: analizar cómo los enfermeros utilizan teorías de enfermería en la sistematización de su práctica clínica. Metodología: revisión integrativa de la literatura, realizada en las bases de datos LILACS, BDENF y SciELO, de donde fueron seleccionados 19 artículos que compusieron la muestra. Resultados: en el 79\% de los artículos analizados se enfatizó la necesidad de utilizar un modelo teórico para implementar el Proceso de Enfermería, siendo las más utilizadas la Teoría de la Adaptación de Callista Roy y la de Jean Watson, ambas utilizadas para la implementación de la Sistematización de la Atención en 15,7\% de la muestra. Entre los ambientes focales de su utilización, se destaca el hospital con el 52\%, la atención primaria con el $21 \%$ y el domicilio con el $15 \%$. Conclusión: a pesar de que la enfermería brasileña es considerada una de las mayores productoras de publicaciones científicas en la actualidad, se contempla aún una baja utilización de teorías de enfermería aliadas a la sistematización de su hacer específico.

Descriptores: Enfermería; Teoría de Enfermería; Proceso de Enfermería.

\section{INTRODUÇÃO}

As teorias de enfermagem consistem num referencial de trabalho que favorece o desenvolvimento do conhecimento científico. Em virtude disso, as teorias de Enfermagem têm sido descritas na literatura como possibilidade de oferecer respaldo científico à prática assistencial, além de proporcionar segurança e qualidade para as ações de enfermagem, uma vez que possibilitam intervenções planejadas passíveis de avaliação para alcance de melhores resultados, possibilitando a visibilidade do trabalho em Enfermagem. ${ }^{1}$

Desde o advento da Enfermagem moderna, através de Florence Nightingale, viu-se a necessidade de aliar a investigação às práticas assistenciais, abrindo caminho para pesquisas visando à organização do conhecimento já produzido. Teorias de conhecimento $^{2}$ têm subsidiado a
Sistematização da Assistência de Enfermagem (SAE), de modo que sua aplicabilidade é feita por meio do processo de enfermagem. Esse é um modelo de processo de trabalho que sistematiza a assistência e direciona o cuidado, permitindo desenvolver atividades que promovam a segurança do usuário e dos profissionais do sistema de saúde. ${ }^{3}$

$O$ ser e o fazer enfermagem necessitam estar apoiados em bases que possibilitem tanto a geração de novas teorias, como a reformulação dos modelos por elas utilizados, potencializando a tomada de decisões e a execução qualificada das ações de enfermagem nos diferentes espaços de inserção profissional. $^{4}$

Ao considerar a combinação da habilidade adquirida durante o processo de formação e a competência despertada a partir da consciência sobre o dever de 
prestar um cuidado qualificado, compreende-se a necessidade de o enfermeiro incorporar a sistematização da assistência ao seu fazer cotidiano. Para isso, existem múltiplos fatores que dificultam a implementação da SAE, relacionando a desvinculação da ação a algum modelo teórico, estando todos ligados apenas a etapa de aplicação do modelo conceitual. ${ }^{5}$ Pode-se até indagar se as demais tentativas de implementação têm procurado seguir algum modelo conceitual ou são realizadas empiricamente, sem um modelo teórico que subsidie essa prática.

Partindo do pressuposto de que aliar a utilização de teorias ao processo de enfermagem para a sistematização da assistência possibilita o desenvolvimento de uma prática qualificada, entende-se a importância de aliar o conhecimento científico à prática assistencial, visando melhoras da assistência, da qualidade das informações, da organização do serviço e dos resultados obtidos.

Considerando esta problemática e a importância do papel desempenhado pelo enfermeiro na assistência, este estudo é norteado pela seguinte pergunta: como os enfermeiros têm utilizado as teorias de enfermagem à sistematização da sua prática clínica? A fim de responder a esse questionamento, este estudo tem como objetivo: identificar na literatura nacional e internacional, estudos que retratem a utilização das teorias de enfermagem na SAE.

\section{METODOLOGIA}

Trata-se de um estudo do tipo revisão integrativa de literatura. Para o desenvolvimento do estudo foram utilizadas as seguintes etapas: estabelecimento da questão norteadora; amostragem ou procedimento para busca na literatura; categorização e avaliação dos estudos incluídos na revisão; interpretação dos resultados e síntese do conhecimento ou apresentação da revisão. ${ }^{6}$

As bases de dados elencadas para identificação dos estudos foram: Literatura Latino-Americana e do Caribe em Ciências da Saúde (LILACS), Bases de dados de enfermagem (BDENF) e Scientific Electronic Library Online (SciELO).

A coleta de dados foi estabelecida no período de dezembro de 2015 a fevereiro de 2016. Na estratégia de busca, utilizaram-se os descritores: (1) - processos de enfermagem, (2) - teoria de enfermagem, de acordo com a terminologia dos Descritores em Ciências da saúde (DeCS), da Biblioteca Virtual em Saúde - (http://decs.bvs.br). Os critérios de inclusão utilizados para a seleção da amostra foram: artigos indexados frutos de pesquisas primárias, disponíveis na íntegra, sobre a utilização das teorias de enfermagem à sistematização da prática clínica do enfermeiro, publicados entre os 
anos de 2011 a 2015. Foram excluídos artigos científicos que não estavam disponíveis na língua portuguesa, artigos sem resumo ou indisponíveis na íntegra on-line e aqueles que não estejam de acordo com a temática.

Ao final da busca, foram identificados quarenta e quatro (44) artigos nas bases de dados LILACS, BDENF e SciELO, sendo realizadas leituras dos resumos, fazendo-se uma triagem quanto à relevância e à propriedade que responderam ao objetivo do estudo. Foram pré-selecionados os artigos científicos analisados quanto às variáveis: ano de publicação; sujeitos da pesquisa; objetivo da pesquisa, e a aplicação da teoria na prática clínica.

Diante dos que correspondiam ao objetivo proposto, foram excluídos 25 por não atenderem aos critérios de inclusão. Ao final, 19 trabalhos compuseram a amostra desta revisão, os quais foram eletronicamente arquivados para a análise e são apresentados logo mais no Quadro 1, segundo ano de publicação, título e seus respectivos autores.

Os dados de cada estudo foram extraídos através de um instrumento elaborado para esta finalidade, o Instrumento para Análise Crítica Externa e Interna e, posteriormente, analisados por meio da estatística descritiva simples.
A análise externa abordou os dados acerca das seguintes variáveis: base de dados de indexação, ano de publicação, língua, origem e periódico. A crítica interna se debruçou sobre os aspectos ligados ao conhecimento, considerando estas variáveis: resultados e conclusões. As informações produzidas foram organizadas com o apoio de tabelas e analisadas de maneira descritiva, através da frequência simples e relativa das variáveis em estudo, sendo agrupadas por temas pertinentes ao assunto para facilitar a compreensão do mesmo.

\section{RESULTADOS}

A partir da busca realizada, 44 publicações foram encontradas nas seguintes bases de dados: 9,09\% na LILACS; $15,9 \%$ no BDENF e $75 \%$ na SciELO. Explica-se que na SciELO não foram encontrados documentos com as estratégias de busca utilizadas para as outras duas bases, sendo necessário ajustar o método de pesquisa. Esses estudos foram selecionados, considerando os critérios estabelecidos, sendo que 19 artigos demonstraram ser contributivos para a elucidação da questão norteadora desta revisão, ressaltando-se que no banco BDENF foram encontradas duas publicações, anteriormente localizadas na base LILACS. 
Quadro 1 - Distribuição dos artigos selecionados segundo ano, título e autores.

\begin{tabular}{|c|c|c|c|}
\hline Ordem & Ano & $\begin{array}{l}\text { Título } \\
\end{array}$ & Autor(es) \\
\hline 1 & 2011 & $\begin{array}{c}\text { Sistematização da Assistência de Enfermagem: } \\
\text { potencialidades reconhecidas pelos enfermeiros de um } \\
\text { hospital público. }\end{array}$ & $\begin{array}{l}\text { KRAUZER, Ivete Maroso; } \\
\text { GELBCKE, Francine Lima }\end{array}$ \\
\hline 2 & 2011 & $\begin{array}{c}\text { Pessoas com úlceras venosas: estudo do modo } \\
\text { psicossocial do Modelo } \\
\text { Adaptativo de Roy. }\end{array}$ & $\begin{array}{l}\text { COSTA, Isabelle Katherinne } \\
\text { Fernandes et al . }\end{array}$ \\
\hline 3 & 2011 & $\begin{array}{l}\text { Da pesquisa à prática de enfermagem aplicando o } \\
\text { modelo de adaptação de Roy. }\end{array}$ & $\begin{array}{c}\text { COELHO, Sónia Margarida } \\
\text { Santos; MENDES, Isabel } \\
\text { Margarida Dias Monteiro. }\end{array}$ \\
\hline 4 & 2012 & $\begin{array}{l}\text { Sistematização da assistência de enfermagem na } \\
\text { perspectiva dos enfermeiros: uma abordagem } \\
\text { metodológica na teoria fundamentada. }\end{array}$ & $\begin{array}{l}\text { MEDEIROS, Ana Lúcia de; } \\
\text { SANTOS, Sérgio Ribeiro dos; } \\
\text { CABRAL, Rômulo Wanderley } \\
\text { de Lima. }\end{array}$ \\
\hline 5 & 2012 & $\begin{array}{c}\text { Competência para prestar cuidado de enfermagem } \\
\text { transcultural à pessoa com deficiência: instrumento de } \\
\text { autoavaliação. }\end{array}$ & $\begin{array}{l}\text { PAGLIUCA, Lorita Marlena } \\
\text { Freitag; MAIA, Evanira } \\
\text { Rodrigues. }\end{array}$ \\
\hline 6 & 2012 & $\begin{array}{l}\text { Análise das respostas comportamentais ao câncer de } \\
\text { mama utilizando o modelo adaptativo de Roy. }\end{array}$ & $\begin{array}{l}\text { SANTOS, Letícia Rosa; } \\
\text { TAVARES, Glaucia Batista; } \\
\text { REIS, Paula Elaine Diniz dos. }\end{array}$ \\
\hline 7 & 2013 & $\begin{array}{c}\text { Assistência de enfermagem a uma puérpera utilizando a } \\
\text { teoria de Horta e a CIPE }\end{array}$ & $\begin{array}{l}\text { LEITE, Maria Clerya Alvino et } \\
\text { al. }\end{array}$ \\
\hline 8 & 2013 & $\begin{array}{l}\text { Cuidado de enfermagem a pessoas com hipertensão } \\
\text { fundamentado na teoria de Parse. }\end{array}$ & $\begin{array}{l}\text { SILVA, Fabíola Vládia Freire } \\
\text { da et al. }\end{array}$ \\
\hline 9 & 2013 & $\begin{array}{l}\text { Teoria do cuidado transpessoal de Jean Watson no } \\
\text { cuidado domiciliar de enfermagem a criança: uma } \\
\text { reflexão. }\end{array}$ & GOMES, Ingrid Meireles et al. \\
\hline 10 & 2013 & $\begin{array}{l}\text { Demandas de cuidado domiciliar da criança nascida } \\
\text { exposta ao HIV na ótica da teoria ambientalista. }\end{array}$ & $\begin{array}{l}\text { LIMA, Ivana Cristina Vieira de } \\
\text { et al. }\end{array}$ \\
\hline 11 & 2013 & $\begin{array}{c}\text { Modelo de atenção crônica: inserção de uma teoria de } \\
\text { enfermagem. }\end{array}$ & $\begin{array}{l}\text { FURTADO, Luciana Gomes; } \\
\text { NOBREGA, Maria Miriam } \\
\text { Lima da. }\end{array}$ \\
\hline 12 & 2013 & $\begin{array}{l}\text { Teoria de Tornar-se Humano na enfermagem } \\
\text { ecológica: aplicando o método de avaliação de Meleis. }\end{array}$ & $\begin{array}{l}\text { LINS, Glauce Araújo Ideião et } \\
\text { al. }\end{array}$ \\
\hline 13 & 2014 & $\begin{array}{l}\text { Consulta coletiva de crescimento e desenvolvimento da } \\
\text { criança à luz da teoria de Peplau }\end{array}$ & $\begin{array}{l}\text { GURGEL, Polyanna Keitte } \\
\text { Fernandes; TOURINHO, } \\
\text { Francis Solange Vieira; } \\
\text { MONTEIRO, Akemi Iwata. }\end{array}$ \\
\hline 14 & 2014 & $\begin{array}{c}\text { Implementação do processo de enfermagem em uma } \\
\text { área da saúde: modelos e estruturas de avaliação } \\
\text { utilizados. }\end{array}$ & $\begin{array}{l}\text { HUITZI-EGILEGOR, Joseba } \\
\text { Xabier et al }\end{array}$ \\
\hline 15 & 2014 & $\begin{array}{l}\text { A prática do cuidado do enfermeiro com famílias de } \\
\text { criança à luz de Jean Watson. }\end{array}$ & $\begin{array}{l}\text { SANTOS, Maiara Rodrigues } \\
\text { dos, et al. }\end{array}$ \\
\hline 16 & 2015 & $\begin{array}{l}\text { Consulta de enfermagem a idosos: instrumentos da } \\
\text { comunicação e papéis da enfermagem segundo Peplau. }\end{array}$ & $\begin{array}{l}\text { SILVA, Juliana Paiva Góes da, } \\
\text { et al. }\end{array}$ \\
\hline 17 & 2015 & $\begin{array}{l}\text { Construção e validação de conteúdo do histórico de } \\
\text { enfermagem guiado pelo referencial de Orem. }\end{array}$ & $\begin{array}{l}\text { DOMINGOS, Camila Santana, } \\
\text { et al. }\end{array}$ \\
\hline 18 & 2015 & $\begin{array}{l}\text { Facilitadores do processo de transição para o } \\
\text { autocuidado da pessoa com estoma: subsídios para } \\
\text { Enfermagem. }\end{array}$ & MOTA, Marina Soares, et al. \\
\hline 19 & 2015 & $\begin{array}{l}\text { Teoria Ambientalista de Florence Nightingale: Uma } \\
\text { Análise Crítica. }\end{array}$ & $\begin{array}{l}\text { MEDEIROS, Ana Beatriz de } \\
\text { Almeida; ENDERS, Bertha } \\
\text { Cruz LIRA; Ana Luisa Brandão } \\
\text { de Carvalho }\end{array}$ \\
\hline
\end{tabular}

Fonte: Autores, 2016. 
A primeira variável analisada foi $\mathrm{o}$ ano de divulgação, em que $31,6 \%$ (06) dos artigos foram publicados no ano de 2013 e os demais divididos entre os anos de 2011, 2012 e 2014 somando respectivamente $15,7 \%$ cada um e 2015 com $21,5 \%$. Todos os 19 trabalhos analisados estavam publicados em português, sendo importante ressaltar que apesar dos trabalhos selecionados serem originários do Brasil, destaca-se um de origem espanhola, proveniente de Gipuzkoa, Província do País Basco, localizado ao Norte da Espanha. Ademais, não foram encontrados trabalhos de outras procedências, provavelmente devido aos critérios de busca utilizados e as características dos bancos de dados consultados.

Analisando-se o veículo de publicação, tem-se que $35,2 \%$ foram publicados na Escola Anna Nery Revista de Enfermagem; $15,7 \%$ da Revista Gaúcha de Enfermagem; 10,5\% da Revista da Escola de Enfermagem da USP; e outros $10,5 \%$ da Rev. Texto \& Contexto Enfermagem. Quanto aos periódicos restantes, identificou-se que pertenciam as revistas: Revista de enfermagem da UFSM; Revista Brasileira de Enfermagem - $\quad$ REBEn; Rev. RENE: Revista da Rede de Enfermagem do Nordeste; Revista Latino-americana de Enfermagem, vinculada a Escola de Enfermagem de Ribeirão Preto da
Universidade de São Paulo - USP; Revista da Escola de Enfermagem da USP e a REME - Revista Mineira de Enfermagem, cada uma com 5,2\% de representatividade, totalizando $31,8 \%$. Tem-se com isso que todos os estudos selecionados foram divulgados em periódicos nacionais e de circulação internacional.

A crítica interna traz os aspectos relacionados ao conteúdo dos artigos. Quanto aos resultados apresentados pelos estudos, foi possível verificar que $79 \%$ enfatizavam a necessidade de utilizar um modelo teórico para implementar o Processo de Enfermagem (PE). Os demais $21 \%$ confirmavam os efeitos da construção e validação de instrumentos de coleta de dados para a consulta de enfermagem, explanando que essa prática oferece subsídios para direcionar os enfermeiros a documentarem a consulta sob um referencial teórico. Esse elo gerado entre a teoria e o instrumento torna-o significativo para o cuidado de enfermagem, facilitando o fornecimento de dados que guiem as decisões clínicas do enfermeiro e colaborem para a implementação de mudanças no fazer da enfermagem.

Ainda sobre a crítica interna dos estudos selecionados, passou-se a análise das conclusões contidas nos documentos, o que permitiu identificar os achados a que chegaram esses estudos acerca da 
sistematização da assistência de enfermagem, onde, sumariamente, todos os estudos estavam voltados para a qualificação do cuidado de enfermagem.

Em $100 \%$ dos estudos, visualizouse uma consonância a respeito da sistematização da assistência de enfermagem constituir-se em um instrumento fundamental no trabalho do enfermeiro, pois possibilita, segundo os autores, visualizar a prática assistencial do profissional e viabiliza a valorização do seu fazer. Dos 19 artigos analisados, 52\% tinham o hospital como ambiente de intervenção para aplicação de um modelo teórico ou da SAE, e seu público em geral variava desde assistência obstétrica nos períodos pré/pós-parto, assistência a estomizados com enfoque nas suas necessidades básicas e seu processo de adaptação, portadores de doenças crônicas, condutas a pessoas com úlceras venosas, e crianças.

Quanto aos estudos que focavam na Unidade de Saúde da Família, foram identificados que $21 \%$ abordavam a visão da assistência à criança na sua consulta de crescimento e desenvolvimento, e as condutas frente à pessoa portadora de
Diabetes Mellitus (DM), aos portadores de Hipertensão Arterial Sistêmica (HAS), além dos cuidados prestados ao público idoso que procura a atenção primária.

Dentre os $15,7 \%$ que focavam no ambiente domiciliar, destacou-se o artigo que caracteriza as condições ambientais desfavoráveis para a saúde das crianças nascidas expostas ao Vírus da Imunodeficiência Humana (HIV). Entretanto, os demais não expunham o local exato para a intervenção, abrangendo toda e qualquer situação em que seja preciso intervir no estado de saúde do usuário.

No que se refere às teoristas utilizadas na argumentação dos artigos, foram identificadas 11 teoristas apresentadas no Quadro 2. Verificou-se que $15,7 \%$ discutiram sobre a Teoria Da Adaptação de Callista Roy; 15,7\% sobre Jean Watson; 10,5\% debatiam sobre Florence Nightingale, com a Teoria Da Manipulação Do Ambiente; além de 10,5\% sobre Hildegard Peplau; 10,5\% abordaram o referencial de Wanda Horta, discutindo sobre o Processo de Enfermagem e a Teoria das Necessidades Humanas Básicas; 10,5\% Afaf Ibrahim Meleis.

Quadro 2 - Distribuição dos artigos encontrados segundo teoristas.

\begin{tabular}{|c|c|}
\hline Teoristas & Qnt \\
\hline Callista Roy & 03 \\
\hline Jean Watson & 03 \\
\hline Afaf Ibrahim Meleis & 02 \\
\hline
\end{tabular}




\begin{tabular}{|c|c|}
\hline Wanda Horta & 02 \\
\hline Florence Nightingale & 02 \\
\hline Hildegard Peplau & 02 \\
\hline Dorothea Elizabeth Orem & 01 \\
\hline Madeleine Leininger & 01 \\
\hline Rosemarie Rizzo Parse & 01 \\
\hline Virginia Henderson & 01 \\
\hline Barney Glaser e Anselm Strauss & 01 \\
\hline Total & $\mathbf{1 9}$ \\
\hline
\end{tabular}

Fonte: Autores; 2016.

Ademais, foram encontrados artigos que discorriam com as seguintes teoristas: Dorothea Elizabeth Orem; Madeleine Leininger; Rosemarie Rizzo Parse e Virginia Henderson, cada um com $5,2 \%$ de representatividade. Um artigo em particular se destacou por sua abordagem, apesar de não tratar diretamente de uma teoria de Enfermagem, a Grounded Theory, ou Teoria Fundamentada nos Dados (TFD). Esta é possível de ser aplicada na pesquisa em enfermagem e foi utilizada por seus autores para operacionalizar a teoria mais adequada ao momento.

\section{DISCUSSÃO}

Tem-se que o termo teoria se refere a uma explicação sistemática de como os fenômenos estão inter-relacionados. Seus principais objetivos são: dar significado aos resultados científicos; resumir o conhecimento existente em sistemas coerentes; estimular novas pesquisas; fornecer direção ao estudo, bem como, explicar a natureza das relações entre as variáveis. As teorias de enfermagem são descritas como alicerces para dar suporte à prática de uma boa assistência, aliadas ao conhecimento específico, estruturado e organizado. $^{7}$

Dentre as teoristas mais utilizadas pelos autores, tem-se a de Callista Roy, que em seu Modelo de Adaptação objetiva contribuir para a saúde, a qualidade de vida e a morte com dignidade. Ela considera como objetivo primordial da enfermagem, a promoção da adaptação dos indivíduos e grupos nos quatro modos de adaptação: físico-fisiológico, identidade de autoconceito, interdependência e desempenho de papel. ${ }^{8}$

A pessoa no Modelo de Adaptação de Roy é um ser social, mental, espiritual e físico, afetado por estímulos do ambiente, sendo o ser humano um sistema biopsicossocial com a capacidade de ajustar-se ao ambiente bem como modificá-lo. ${ }^{9}$

Nesse sentido, o cuidado de enfermagem, visualizado a partir da ótica da teoria da adaptação de Roy, direciona as 
ações de enfermagem de forma a abranger a pessoa que recebe o cuidado considerando-a no todo em suas relações com ambiente interno e externo. ${ }^{4}$ As metas visam ao restabelecimento e/ou à manutenção do equilíbrio sendo possível relacioná-las às intervenções necessárias. Portanto, trata-se de um sistema adaptativo holístico, em contínua interação com o meio ambiente mutante.

A segunda de maior destaque foi a Teoria do Cuidado Humano de Jean Watson, a qual está centrada no conceito de cuidado e em pressupostos fenomenológicos existenciais, que traz o olhar para além do corpo físico. A teoria desenvolvida por Watson ressalta a enfermagem como a ciência do cuidado ${ }^{10}$, desde que baseada em paradigmas metafísicos, filosóficos e morais, respeitando o ser humano em sua unidade e totalidade de mente-corpo-espírito oferecendo um alicerce para a profissão e para o cuidar.

Existem discordâncias entre autores $^{11}$ a respeito da adequada utilização da fundamentação teórica de Watson pelo profissional enfermeiro. Os conceitos da teoria são pouco reconhecidos na prática do enfermeiro. Isso reflete diretamente na execução da assistência, sendo preciso uma consciência clara sobre a importância de aprimorar o conhecimento e ter o respaldo de um referencial teórico para a enfermagem, reconhecendo a pertinência de articular os elementos da teoria de Jean Watson na prática.

A teoria ambientalista de Florence Nightingale, a qual foi descrita em dois (02) dos estudos analisados, abrange três relações principais: ambiente com o doente; enfermeira com o ambiente e enfermeira com o doente. Considera o ambiente como o fator principal que atua sobre o indivíduo gerando um estado de doença. A precursora da enfermagem, com vistas nas relações entre o ser humano, ambiente e natureza, trouxe consigo em sua época, incontáveis contribuições para a melhoria e o desenvolvimento da saúde, mantendo-se, até os dias atuais, como fonte de inspiração e alvo de pesquisa para estudiosos em todo o mundo. ${ }^{12,13}$

Para Peplau o enfermeiro deve usar os instrumentos da comunicação: escuta, esclarecimento e aceitação, e que a enfermagem exerce pelo menos seis papéis fundamentais: estranho, provedor de recursos, professor, líder, substituto e assessor. $^{14}$

Observa-se que as teorias quase sempre têm princípios comuns. A exemplo disso, não tão distante da realidade proposta por Orem, está a Teoria da Diversidade e Universalidade do Cuidado Cultural (TDUCC) de Madeleine Leininger, que busca uma assistência, onde 
o ser cuidado tenha autonomia, liberdade e poder para tomada de decisão. ${ }^{15}$

Dentre as teoristas descritas nos estudos e que indicam o estímulo pelo enfermeiro ao protagonismo do sujeito em seu processo de tratamento, identifica-se um estudo sobre Rosemarie Rizzo Parse, a qual considera o ser humano de forma individual e possuidor de livre arbítrio. $\mathrm{O}$ enfermeiro aparece como mediador e colaborador no processo de recuperação ou manutenção de um tratamento. ${ }^{16}$

Percebeu-se uma maior utilização de teorias de enfermagem atrelada ao ambiente hospitalar. Onde, para a elaboração e implementação de um modelo assistencial em um serviço hospitalar, é necessária a realização de um diagnóstico do trabalho da equipe de enfermagem ${ }^{17}$, ressaltando seu preparo técnico-científico sobre o processo de enfermagem, bem como a existência de problemas decorrentes de uma assistência não sistematizada.

Os enfermeiros autores dos estudos em análise compreendem a sistematização da assistência de enfermagem como um método de trabalho derivado do método científico. A SAE desenvolve-se através de uma assistência que valoriza a enfermagem, melhora a qualidade da assistência prestada, e contribui para o aprendizado por se apoiar em marcos teóricos e filosóficos que necessitam ser estudados e discutidos entre os enfermeiros. $^{18}$

Nesse processo de reestruturação do processo de cuidar em enfermagem, é relevante o papel do enfermeiro, que precisa refletir sobre a necessidade de incrementar assistência com ferramentas que norteiem a prática de enfermagem de forma segura, organizada e competente, em que se destaca a utilização do processo de enfermagem, baseada em um modelo teórico, apropriado por meio da sistematização da assistência de enfermagem. ${ }^{19}$

Apesar do crescente interesse da comunidade acadêmica por identificar melhorias na qualidade da assistência prestada ao usuário, e os autores encontrados referirem a inserção das teorias de enfermagem na prática clínica, este estudo apresenta limitações como a existência de poucas publicações a respeito do uso das teorias de enfermagem na sistematização da assistência do enfermeiro no Brasil. Outra limitação identificada está relacionada ao fato de terem sido utilizados somente os artigos disponíveis nas bases de dados eletrônicas, em língua portuguesa, o que pode ter contribuído, para que outros trabalhos também relevantes não tenham sido acessados e talvez, incluídos a pesquisa.

\section{CONCLUSÃO}


O estudo permitiu apreender que, apesar de a enfermagem brasileira ser considerada uma das maiores produtoras de publicações científicas na atualidade, contempla-se ainda uma baixa utilização de teorias de enfermagem aliadas à sistematização do seu fazer específico, refletindo a influência do contexto social e político no qual está imersa a profissão, bem como seu distanciamento de reflexões sobre seus aspectos ontológicos, sobre os quais tem pouco se debruçado, em detrimento dos epistemológicos.

Verificou-se preocupação dos profissionais que prestam assistência com a utilização de teorias de enfermagem, agarrando-se a isso como um esforço para a valorização das mesmas, objetivando a organização do trabalho, com uso de uma linguagem própria da profissão e com a produção de novos conhecimentos, sendo este, um processo de amadurecimento gradativo que vêm desde a formação acadêmica até sua atuação enquanto profissional.

Considerando a Enfermagem como prática social e que grande parte das teoristas da área de Enfermagem situa a atuação do enfermeiro vinculada à atenção das necessidades humanas, este estudo traz como provocação para a reflexão dos próprios enfermeiros e demais membros da equipe de enfermagem a metodologia utilizada na construção da sistematização da assistência.

Estejamos atentos às implicações com as quais se atrelam o nosso fazer específico e, acima de tudo, engajemo-nos para uma crítica à própria profissão debruçando-nos sobre os aspectos sócio históricos determinantes para a conjuntura atual, e consequentemente na luta para possibilitar a comunidade usuária do sistema de saúde uma atenção de enfermagem digna e de qualidade, pois apenas alcançaremos um sistema mais justo se nos envolvermos e nos comprometermos com as transformações necessárias.

\section{REFERÊNCIAS}

1. Matos JC, Luz GS, Marcolino JS, Carvalho MDB, Pelloso SM. Ensino de teorias de enfermagem em Cursos de Graduação em Enfermagem do Estado do Paraná - Brasil. Acta paul. Enferm. 2011;24(1):23-28.

2. Medeiros AL, Santos SR, Cabral RWL. Sistematização da assistência de enfermagem na perspectiva dos enfermeiros: uma abordagem metodológica na teoria fundamentada. Rev. Gaúcha Enferm. 2012;33(3):174-181.

3. Menezes SRT, Priel MR, Pereira LL.

Autonomia e vulnerabilidade do enfermeiro na prática da Sistematização da Assistência de Enfermagem. Rev. Esc. enferm. USP. 2011;45(4):953-958.

4. Costa IKF, Nóbrega WG, Costa IKF, Torres GV, Lira ALBC, Tourinho FSV, et al. Pessoas com úlceras venosas: estudo do modo psicossocial do modelo adaptativo de Roy. Rev. Gaúcha Enferm. 2011;32(3):561-568. 
5. Hermida PMV. Desvelando a implementação da Sistematização da Assistência de Enfermagem. Rev. bras. enferm. 2004;57(6):733-737.

6. Mendes KDS, Silveira RCCP, Galvão

CM. Revisão integrativa: método de pesquisa para a incorporação de evidências na saúde e na enfermagem. Texto contexto - enferm. 2008;17(4):758-764.

7. Raimondo ML, Fegadoli D, Méir MJ, Wall ML, Labronici LM, Ferraz-Raimondo ML. Produção científica brasileira fundamentada na Teoria de Enfermagem de Orem: revisão integrativa. Rev. bras. enferm. 2012;65(3):529-534.

8. Coelho SMS, Mendes IMDM. Da pesquisa à prática de enfermagem aplicando o modelo de adaptação de Roy. Esc. Anna Nery. 2011;15(4):845-850. 9. Santos LR, Tavares GB, Reis PED. Análise das respostas comportamentais ao câncer de mama utilizando o modelo adaptativo de Roy. Esc Anna Nery Rev Enferm. 2012;16(3):459-465.

10. Gomes IM, Lima KA, Rodrigues LV, Lima RAG, Collet N. Teoria do cuidado transpessoal de Jean Watson no cuidado domiciliar de enfermagem à criança: uma reflexão. Esc Anna Nery Rev Enferm. 2013;17(3):555-561.

11. Santos MR, Bousso RS, Vendramim P, Baliza, MF, Misko MD, Silva L. A prática do cuidado do enfermeiro com famílias de criança à luz de Jean Watson. Rev. esc. enferm. USP. 2014;48(suplementar):80-86. 12. Lopes LMM, Santos SMP. Florence Nightingale: apontamentos sobre a fundadora da Enfermagem Moderna. Rev. Enf. Ref. 2010; serIII (2):181-189.
13. Frello AT, Carraro TE. Contribuições de Florence Nightingale: uma revisão integrativa da literatura. Esc Anna Nery Rev Enferm. 2013; 17( 3 ): 573-579. 14. Silva JPG, Costa KNFM, Silva GRF, Oliveira SHS, Almeida PC, Fernandes MGM. Consulta de enfermagem a idosos: instrumentos da comunicação e papéis da enfermagem segundo Peplau. Esc. Anna Nery. 2015;19(1):154-161.

15. Seima MD, Michel T, Méier MJ, Wall ML, Lenardt MH. A produção científica da enfermagem e a utilização da teoria de Madeleine Leininger: revisão integrativa 1985-2011. Esc Anna Nery Rev Enferm. 2011;15(4):851-857.

16. Silva FVF, Silva LF, Guedes MVC, Moreira TMM, Rabelo ACS, Ponte KMA. Cuidado de enfermagem a pessoas com hipertensão fundamentado na teoria de Parse. Esc Anna Nery Rev Enferm. 2013;17(1):111-119.

17. Andrade JS, Vieira MJ. Prática assistencial de enfermagem: problemas, perspectivas e necessidade de sistematização. Rev. bras. enferm. 2005;58(3):261-265.

18. Krauzer IM, Gelbke FL.

Sistematização da Assistência de Enfermagem: potencialidades reconhecidas pelos enfermeiros de um hospital público. Rev. enferm. UFSM. 2011;1(3):308-317. 19. Furtado LG, Nobrega MML. Modelo de atenção crônica: inserção de uma teoria de enfermagem. Texto \& Contexto enferm. 2013;22(4):1197-1204.

RECEBIDO: $15 / 01 / 2018$

APROVADO: 01/05/2018

PUBLICADO: 07/2018 